\title{
Dynamic Response Modeling of MEMS Micromirror Corner Cube Reflectors With Angular Vertical Combdrives
}

\author{
Young Ki Hong and Richard R. A. Syms, Senior Member, IEEE
}

\begin{abstract}
A model for the dynamic response of microelectromechanical-systems micromirrors with angular vertical combdrive electrostatic actuators has been proposed based on a numerical solution of the nonlinear dynamic equations for torsional oscillation. The torque generated by the electrostatic drive is assumed to depend at least on the second order of the turn angle, and the model shows a shift in the resonant frequency with drive voltage, in agreement with the experimental results obtained from components fabricated by self-assembly of bonded silicon parts. The dynamic response of a corner cube reflector $(\mathrm{CCR})$ based on a pair of scanning mirrors and a fixed mirror is predicted using the model, and the theoretical response agrees well with the experimental results. From the model, a small initial misalignment of the CCR mirrors can be determined.
\end{abstract}

Index Terms-Angular vertical combdrive, corner cube reflector (CCR), microelectromechanical systems (MEMS), micromirror, nonlinear dynamics.

\section{INTRODUCTION}

$\mathbf{E}$ LECTROSTATIC combdrives have been widely used for the actuation of microelectromechanical systems (MEMS) devices. Depending on the electrode geometry, combdrives can be used for linear or angular motion. The former type is called an in-plane combdrive, whereas the latter type is a vertical drive. Compared to a parallel-plate actuator [1], combdrives have the advantages of a larger working range and high stability.

Vertical combdrives have recently been the subject of considerable attention due to their high force densities [2]-[6]. They can be further classified into two types depending on the geometry: The first is a staggered vertical combdrive, which has a vertical asymmetry between the fixed and moving electrodes, in terms of both the depth and position of the fingers [2]-[4], [6]. The second is an angular vertical drive, in which the moving fingers are initially skewed with respect to the fixed fingers [5], [7], [8].

Hah et al. have shown that angular vertical combdrives can offer better performance than staggered vertical drives [8]. However, their behavior is generally difficult to predict because of their inherent nonlinearity [9]-[11]. Xie et al. have analyzed the electrostatic force generated by an angular vertical drive using parallel-plate approximation to calculate the overlap

Manuscript received May 15, 2006; revised September 21, 2006.

Y. K. Hong is with the Samsung Semiconductor R\&D Institution, Seoul 100-742, Korea.

R. R. A. Syms is with the Optical and Semiconductor Devices Group, Department of Electrical and Electronic Engineering, Imperial College London, SW7 2BT London, U.K. (e-mail: r.syms@ic.ac.uk).

Digital Object Identifier 10.1109/JLT.2006.889137 area between the moving and fixed fingers [12]. Although the method correctly estimated the complicated aerial overlap variation between the fingers, the model ignored the fringing field. Hah et al. carried out a similar calculation considering the fringe field. However, in this model, only simple electrode geometries were considered [8].

In this paper, we present a simple numerical model for the nonlinear dynamics of resonant scanning micromirrors with angular vertical combdrives. To develop the model, we first carry out experiments using self-assembled mirror scanners. Using these preliminary data, the equations of motion can be solved regardless of the geometry or fringe-field consideration. The model predictions are in good agreement with the experimental result. Moreover, the response of more complicated devices based on scanning mirrors [for example, modulatable corner cube reflectors (CCRs)] can be predicted using an extended model.

The fabrication of and experimental results obtained from a scanning mirror and a CCR formed by self-assembly of bonded silicon parts are summarized in Section II. A general solution method for the dynamic equations of motion for a torsional oscillator is proposed in Section III. Numerical predictions for the response of a single scanning mirror are presented in Section IV, and the performance of a modulatable CCR based on multiple scanning mirrors and predicted by an extended model is compared with the experimental results in Section V. Conclusions are presented in Section VI.

\section{COMPONEnt FABricAtion, Design, AND OpticAl PERFormance}

Scanning micromirrors and CCRs with angular vertical combdrive actuators were fabricated using a surface tension self-assembly method previously described in [7], [9], and [13]. In this section, we briefly summarize their fabrication and performance.

\section{A. Fabrication and Design}

Fabrication involved a simple two-mask process:

1) patterning of fixed and movable parts on a bonded siliconon-insulator (BSOI) substrate by optical lithography and deep reactive ion etching;

2) patterning of thick pads of photoresist linking the fixed and movable parts;

3) sacrificial etching of the underlying oxide; 


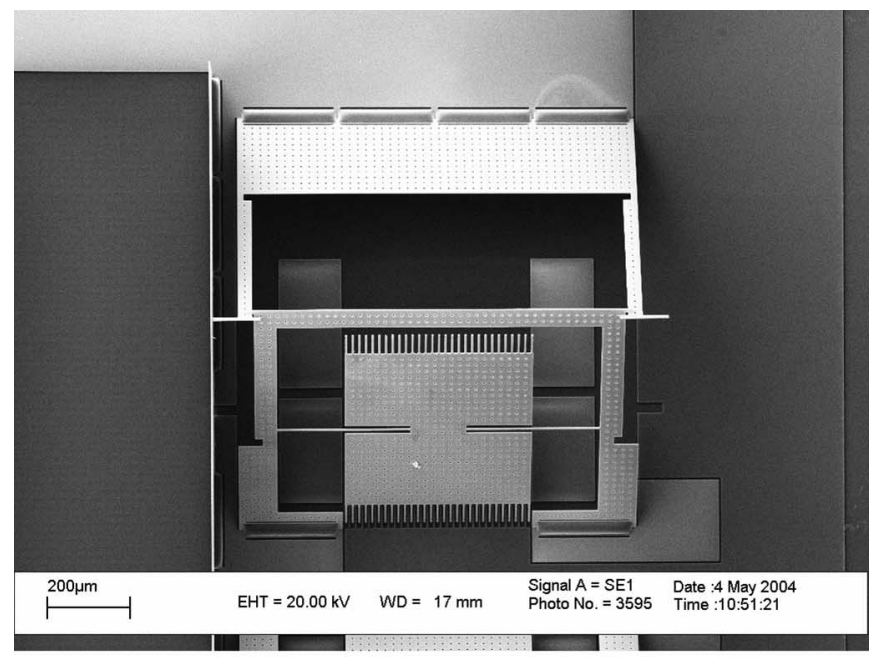

(a)

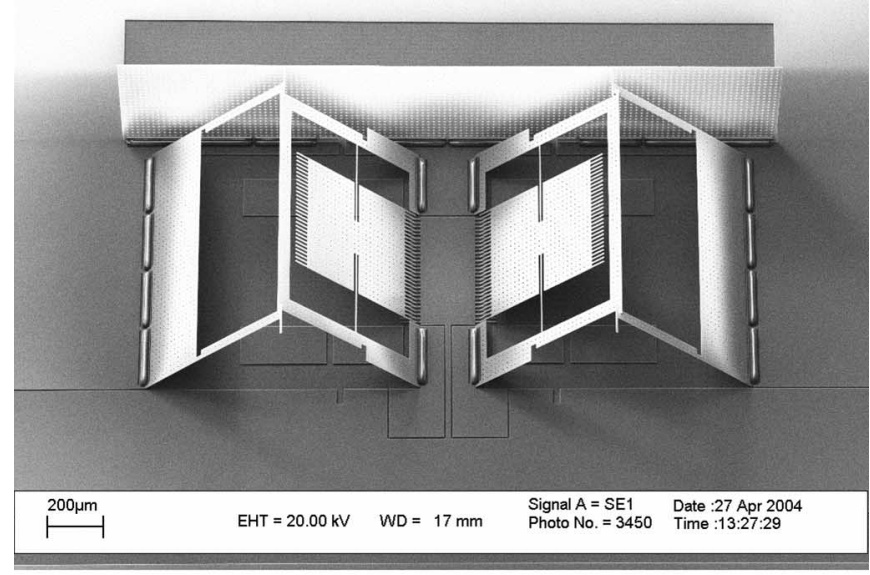

(b)

Fig. 1. Scanning electron microscope view of (a) scanning micromirror and (b) active CCR.

4) freeze-drying to remove wash water without sacrificial layer collapse;

5) out-of-plane rotation of the movable parts by melting the photoresist;

6) sputter coating with $\mathrm{Cr} / \mathrm{Au}$ to allow electrical contact and improve reflectivity.

Fig. 1(a) shows a scanning electron microscope view of a completed micromirror formed in BSOI material with a bonded layer thickness of $3.5 \mu \mathrm{m}$ and an interlayer oxide thickness of $2 \mu \mathrm{m}$. The mirror itself is a rectangular element measuring $496 \times 456 \mu \mathrm{m}$ that is suspended on a torsion bar measuring $5 \times 324 \mu \mathrm{m}$. The mirror is supported by a frame that has been rotated out of plane by the surface tension developed during the self-assembly step and latched in place at an angle of $45^{\circ}$ by a second similarly rotated component. The pads powering the rotation were formed in Shipley AZ4562 photoresist and measure $32 \times 230 \mu \mathrm{m}$, with a thickness of $12 \mu \mathrm{m}$. Melting was carried out in a convection oven at $160^{\circ} \mathrm{C}$. The moving half of an angular vertical drive is carried by the mirror, while the fixed half is provided on a land attached to the substrate. There are 29

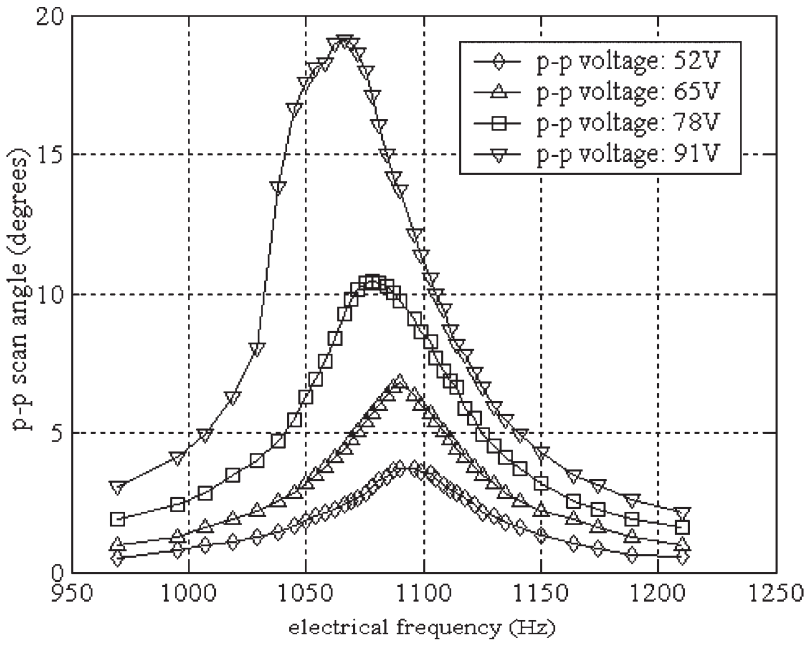

(a)

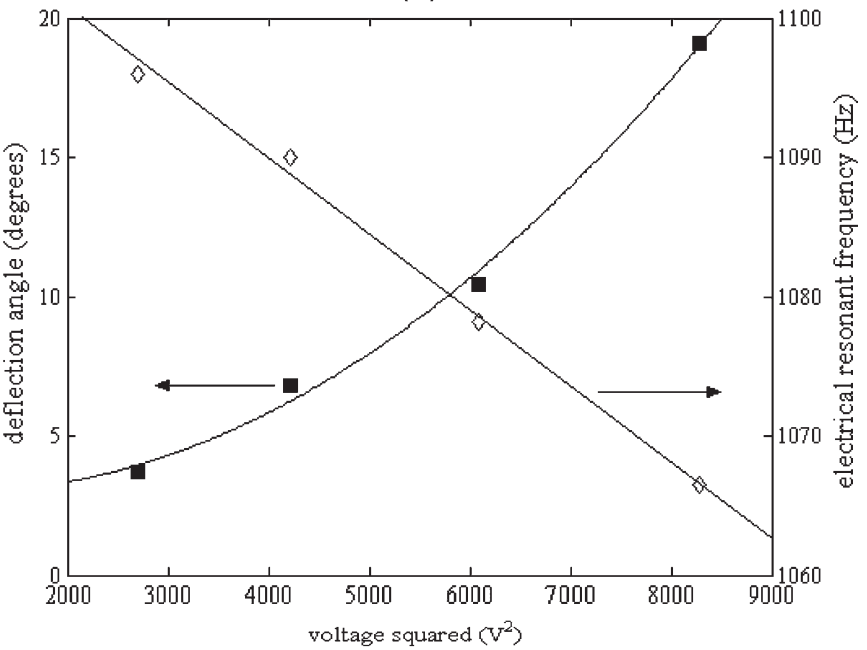

(b)

Fig. 2. (a) Variation of scan angle with frequency at different voltages, for a mirror actuated by an angular vertical combdrive. (b) Variation of scan angle and electrical resonant frequency with square voltage.

moving electrode fingers and 28 fixed fingers. Electrical contact is provided by sputtered metallization, which consisted of $100 \AA \mathrm{Cr}$ and $500 \AA \mathrm{Au}$ metal.

Fig. 1(b) shows a scanning electron microscope view of a CCR based on two $45^{\circ}$ scanning micromirrors and one $90^{\circ}$ fixed mirror. By intermittently changing the angle of either or both of the scanning mirrors, the device can modulate an incident signal and hence transmit data back to a light source [1]. The dimensions of each scanning mirror are similar to those in Fig. 1(a).

\section{B. Optical Performance}

Optical performance was measured by the laser reflection method, as described in [9]. Fig. 2(a) shows the variation of the peak-to-peak scan angle of a scanning mirror with drive frequency, at different drive voltages. These data highlight one aspect of the nonlinear dynamic behavior of the overall system: The resonant frequency clearly decreases as the voltage increases [Fig. 2(b)]. This nonlinear response has been 


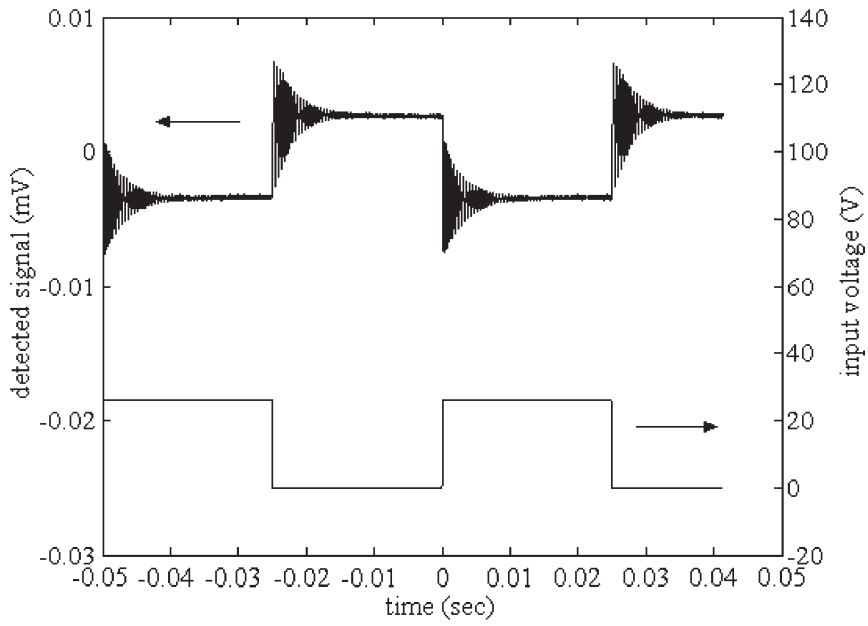

(a)

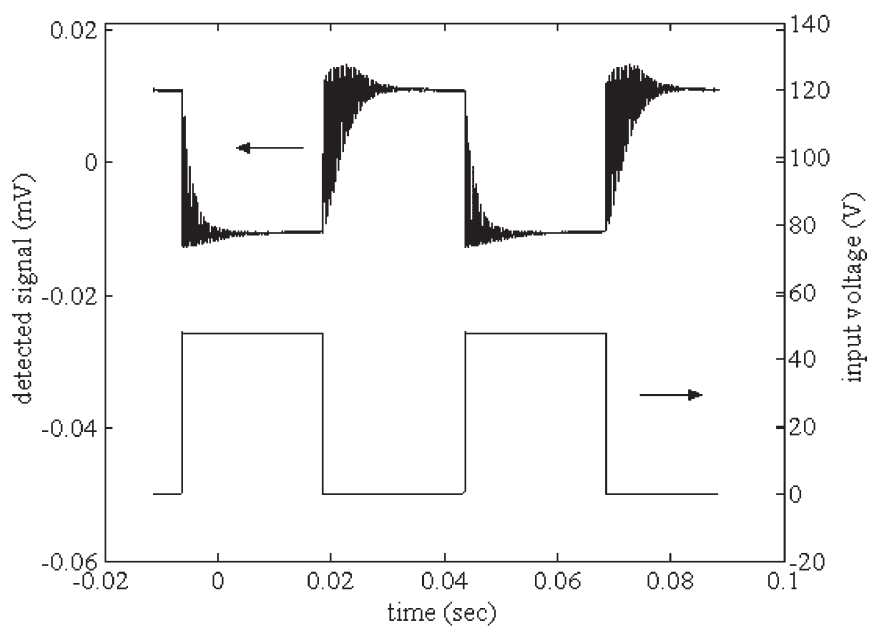

(b)

Fig. 3. Time variation of the retroreflected signal obtained with square-wave voltages of (a) $26 \mathrm{~V}_{\mathrm{p}-\mathrm{p}}$ and (b) $48 \mathrm{~V}_{\mathrm{p}-\mathrm{p}}$.

observed in other devices with angular vertical drives [7], [10], [11], and we present a more detailed theoretical model of this aspect in Section III.

When two scanning mirrors are used as components of a CCR, the modulated signal again varies considerably, depending on the driving voltage. Fig. 3(a) shows the response of an active CCR obtained using a $26 \mathrm{~V}_{\mathrm{p}-\mathrm{p}}$ square wave at a frequency of $20 \mathrm{~Hz}$. Fig. 3(b) shows the response at a higher driving voltage of $48 \mathrm{~V}_{\mathrm{p}-\mathrm{p}}$. Although the signal is effectively modulated in both cases, the transient oscillations at turn-on and turn-off are entirely different. Zhu et al. have previously proposed an optical model for the static response of a CCR [14]. In Section IV, we show how this model may be combined with the solution for the motion of the scanning mirror to predict dynamic behavior.

\section{General Solution Method}

In any electrostatic actuator, the driving force or torque is important. For an in-plane combdrive, the driving force $F$ can be expressed as follows:

$$
F=n t \varepsilon_{0} V^{2} g .
$$

Here, $n$ is the number of electrode fingers, $t$ is their thickness, $\varepsilon_{0}$ is the dielectric constant of free space $\left(8.85 \times 10^{-12} \mathrm{Fm}^{-1}\right)$, $g$ is the gap between the electrode fingers, and $V$ is the applied voltage. For an angular vertical combdrive, it is difficult to obtain an analytic expression for the torque because it must depend on the relative angular position of the electrodes. However, numerical methods can be used to evaluate the torque, as will be shown.

The basic equation of motion for a torsional oscillator is

$$
d^{2} \theta / d t^{2}+2 \zeta \omega_{r} d \theta / d t+\omega_{r}^{2} \theta=T(\theta) / I .
$$

Here, $\theta$ is the turn angle, $T(\theta)$ is the driving torque, $\omega_{r}=$ $(k / I)^{1 / 2}$ is the angular resonant frequency, where $k$ is the angular stiffness and $I$ is the moment of inertia, and $\zeta$ is the damping coefficient. For an electrostatically driven system, the torque is always given by the general expression

$$
T(\theta)=1 / 2 \partial C / \partial \theta V^{2} .
$$

Here, $V$ is the voltage, and $C$ is the electrode capacitance. In general, we may write the variation of the capacitance near a given angle $\theta_{0}$ as a power series of the form

$$
\partial C / \partial \theta=c_{0}+c_{1}\left(\theta-\theta_{0}\right)+c_{2}\left(\theta-\theta_{0}\right)^{2} \ldots
$$

Assuming that $\theta$ is measured from the rest position, we may set $\theta_{0}=0$. Here, constants $c_{0}, c_{1}, c_{2}$, etc. may be found by differentiating the angular variation of the capacitance and fitting a power series to the result. For an angular vertical combdrive, $\partial \mathrm{C} / \partial \theta$ may initially increase with angle, until the moving comb passes through the fixed comb. In the initial phase, only a small positive value of $c_{1}$ needs be considered.

With a sinusoidal excitation, we may write $V=V_{0} \cos (\omega t)$, where $\omega$ is the angular frequency, so that $V^{2}=V_{0}^{2}\{1+$ $\cos (2 \omega t)\} / 2$. Combining (2)-(4) and retaining terms only up to $c_{1}$, we obtain

$$
\begin{aligned}
& d^{2} \theta / d t^{2}+2 \zeta \omega_{r} d \theta / d t+\left\{\omega_{r}^{2}-c_{1} V_{0}^{2} / 4 I\right\} \theta \\
& =\left(c_{0} V_{0}^{2} / 4 I\right)\{1+\cos (2 \omega t)\}+\left(c_{1} V_{0}^{2} \theta / 4 I\right) \cos (2 \omega t) .
\end{aligned}
$$

Note that the effective angular resonant frequency $\omega_{\text {re }}$ in (5) has now become

$$
\omega_{\mathrm{re}}^{2}=\omega_{r}^{2}-c_{1} V_{0}^{2} / 4 I
$$

Using a binomial approximation, we obtain $\omega_{\text {re }}=\omega_{r}-$ $c_{1} \mathrm{~V}_{0}^{2} / 8 I \omega_{r}$. This result suggests that the resonant frequency is modified electrostatically, as previously noted by other authors [11].

To solve (5) numerically, we used the Runge-Kutta method, which is widely accepted as an efficient method of solving firstorder differential equations. The algorithm first computes four 
auxiliary quantities to solve a first-order differential equation $y^{\prime}=f(x, y)$ [15]. The truncation error is of the order of $h^{5}$, where $h$ is the step length, and the method is of the fourth order. ODE45, which is a function of the commercial software package MATLAB 6.0, can implement the Runge-Kutta method using (4) and (5) [16]. Since (5) is of the second order, it is first expressed in terms of two coupled first-order equations in terms of new variables $\theta_{1}=\theta$ and $\theta_{2}$ of the form

$$
\begin{aligned}
\theta_{1}^{\prime}= & \theta_{2} \\
\theta_{2}^{\prime}= & \left(c_{0} V_{0}^{2} / 4 I\right)\{1+\cos (2 \omega t)\} \\
& +\left[\left(c_{1} V_{0}^{2} / 4 I\right)\{1+\cos (2 \omega t)\}-\omega_{r}^{2}\right] \theta_{1}-2 \zeta \omega_{r} \theta_{2} .
\end{aligned}
$$

The ODE45 function is then used to solve (7) and (8) simultaneously.

As a first step in solving the equation, the damping factor $\zeta$ must be known. This term can be determined experimentally from the resonance curve of the mirror as [17]

$$
\zeta=\left(\omega_{2}-\omega_{1}\right) / 2 \omega_{r}
$$

Here, $\omega_{1}$ and $\omega_{2}$ are the angular frequencies at which the oscillation amplitude falls to $1 / \sqrt{ } 2$ of its peak value. We can obtain the damping factor from Fig. 2(a). Since the resonance curve shows nonlinear behavior at a large amplitude, the damping factor is determined from the data set with the smallest amplitude, as $\zeta=0.02$.

The second step is to define the limiting resonant frequency $\omega_{r}$ (i.e., the resonant frequency for small amplitude oscillations) and to choose suitable coefficient values. The analytic approximation of the effective resonant frequency (6) can be used for this purpose. Using the data set used for Fig. 2(b), the limiting resonant frequency can be extrapolated to $2236 \mathrm{~Hz}$, and coefficient $c_{1}$ can be estimated as $4.82 \times 10^{-13}$. To choose a suitable range for $c_{0}$, (5) can be further approximated by assuming that $\theta=\phi_{0}+\phi_{1} \exp (j \omega t)+\phi_{2} \exp (2 \mathrm{j} \omega t)+\cdots$. Substituting this expression into (5) and expanding, we can obtain an approximation given as follows:

$$
\begin{aligned}
\phi_{0} \omega_{r}^{2} & =c_{0} V_{0}^{2} / 4 I+c_{1} \phi_{0} V_{0}^{2} / 4 I \\
\phi_{0} & =c_{0} V_{0}^{2} /\left\{4 I\left(\omega_{r}^{2}-c_{1} V_{0}^{2} / 4 I\right)\right\} .
\end{aligned}
$$

Assuming that $c_{1}=4.82 \times 10^{-13}$ and using the measured data set, the coefficient $c_{0}$ can be estimated as $1.65 \times 10^{-11}$. Equations (7) and (8) can then be solved by varying coefficients $c_{0}$ and $c_{1}$ around these initial values. The boundary conditions are taken as $\theta_{1}=0$ and $\theta_{2}=0$ at $t=0$.

A least square fitting method is used to determine the best values of $c_{0}$ and $c_{1}$. The correctness of the simulation is estimated using a coefficient of determination $R^{2}$, which is an indicator from 0 to 1 that reveals how closely the estimated values for the trend line correspond to the actual data. The coefficient can be expressed as [18]

$$
R^{2}=1-\left\{\sum_{i=0}^{N}\left(y_{i}-Y_{i}\right)^{2}\right\} /\left\{\sum_{i=0}^{N} y_{i}^{2}-\left(\sum_{i=0}^{N} y_{i}\right)^{2} / N\right\}
$$

Here, $y_{i}$ and $Y_{i}$ are a set of actual and predicted values, and $N$ is the number of data points. The simulation result is most reliable when the $R^{2}$ value is at or near 1 . Equation (2) can obviously be solved for higher order approximations to the capacitance variation by substituting more of the terms in (4) into (5). Using this method, the torque on the mirror and the overall dynamic response can then be determined.

\section{SCANning Mirror Response}

Fig. 4 shows the predicted turn-on response of a single mirror, which was obtained using the coefficient values $c_{0}=$ $0.21 \times 10^{-13}, c_{1}=4.79 \times 10^{-13}$, and $c_{2}=43.31 \times 10^{-13}$, for different applied voltages. The drive frequency is assumed to be $1067 \mathrm{~Hz}$. Because the torque is proportional to the square voltage, the period of the resulting oscillation is $1 /(2 f)=$ $0.46 \mathrm{~ms}$. Fig. 4(a) shows the response at a low applied voltage. The magnitude of the oscillation initially increases, then slightly decreases, and finally stabilizes, as expected from a linear device. At a higher voltage, the oscillation amplitude increases for a longer time and stabilizes, as shown in Fig. 4(b). At a very high voltage, the oscillation amplitude increases with time, as shown in Fig. 4(c), and eventually becomes infinite. This result shows the nonlinear response because the electrical torque term in (5) is expressed in combination of both angle and time.

The nonlinear response can also be seen from the detailed shape of the oscillation. Fig. 5 shows the steady-state response obtained with various applied voltages. At a low voltage, the initial shape of the oscillation, which is symmetric with a period of $0.46 \mathrm{~ms}$, is still preserved after a long time, as shown in Fig. 5(a). This period is the same as that of the driving torque. Fig. 5(b) shows the shape at a higher voltage. The period becomes longer, $0.47 \mathrm{~ms}$ in this case, and the symmetric shape of the response starts to disappear. Finally, at a very high voltage, the periodic response after $10 \mathrm{~ms}$ shows a nonsymmetric and unstable shape, and a period of $0.49 \mathrm{~ms}$, as shown in Fig. 5(c). The nonlinear behavior of the angular vertical combdrive therefore alters both the oscillation period and the harmonic content.

The frequency response of the mirror can be evaluated by calculating the stabilized amplitude from the time response obtained at different drive frequencies. By comparing these numerical predictions with real data, the coefficients describing the variation of the capacitance in (4) can be determined.

Fig. 6 shows the least square fitted result, and Table I shows the coefficients obtained using the data sets corresponding to the voltages of 52 and $92 \mathrm{~V}$. Fig. 6(a) shows the model prediction obtained from a first-order approximation for the torque. Although the graph correctly shows the shift of the 


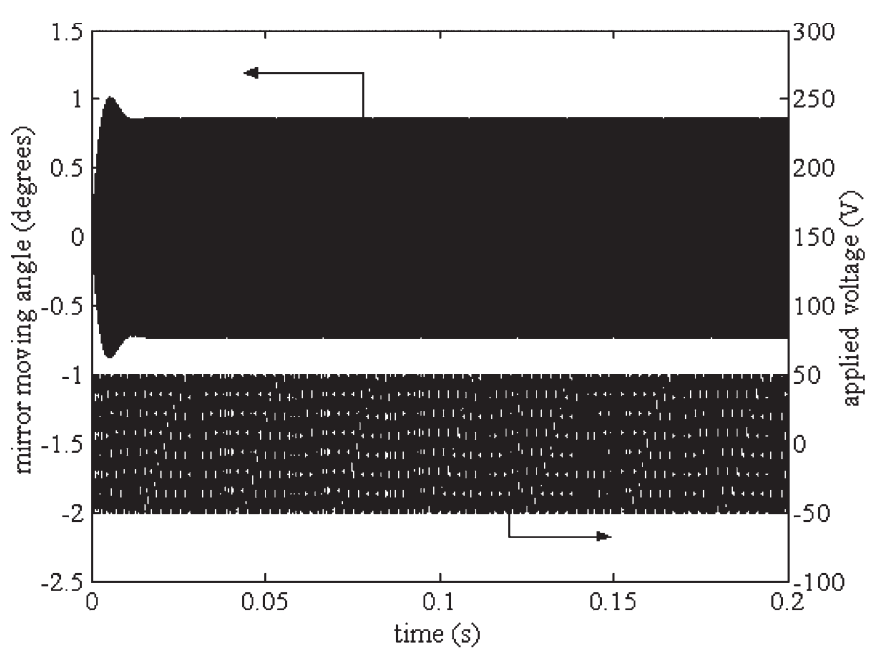

(a)

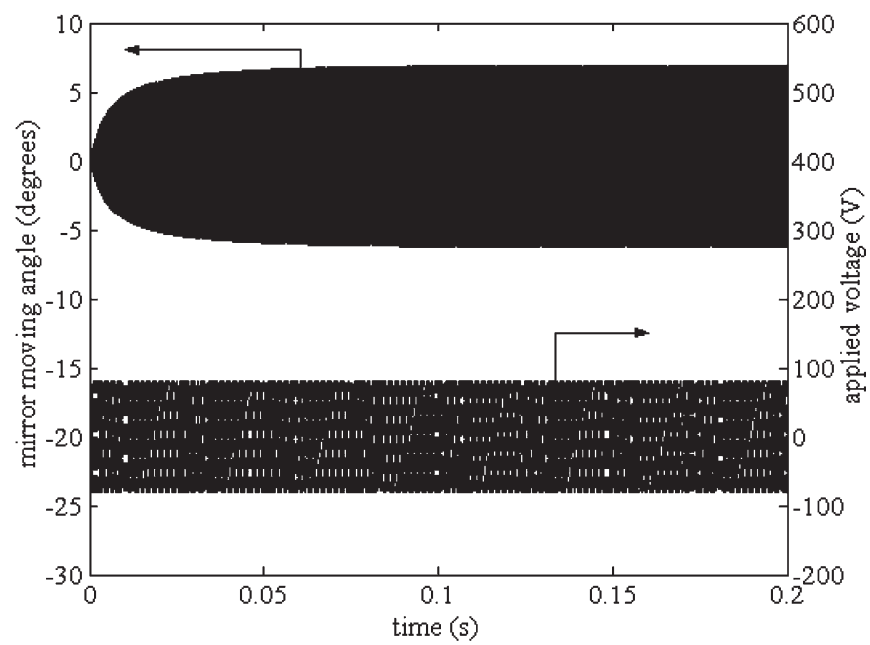

(b)

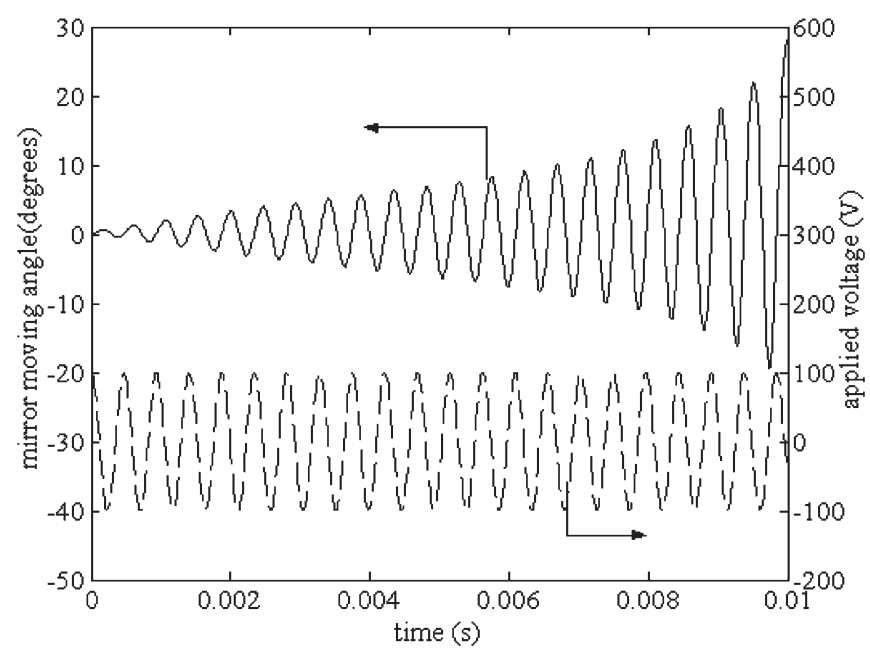

(c)

Fig. 4. Numerically simulated variation of mirror angle with time, which was obtained using coefficients $c_{0}, c_{1}$, and $c_{2}$ and applied sinusoidal voltages of (a) $50 \mathrm{~V}_{\mathrm{p}-\mathrm{p}}$, (b) $80 \mathrm{~V}_{\mathrm{p}-\mathrm{p}}$, and (c) $100 \mathrm{~V}_{\mathrm{p}-\mathrm{p}}$.

resonant frequency with voltage, the absolute angles do not coincide very well. Moreover, in the simulated trend, we cannot see the distortion near the resonant frequency shown in the

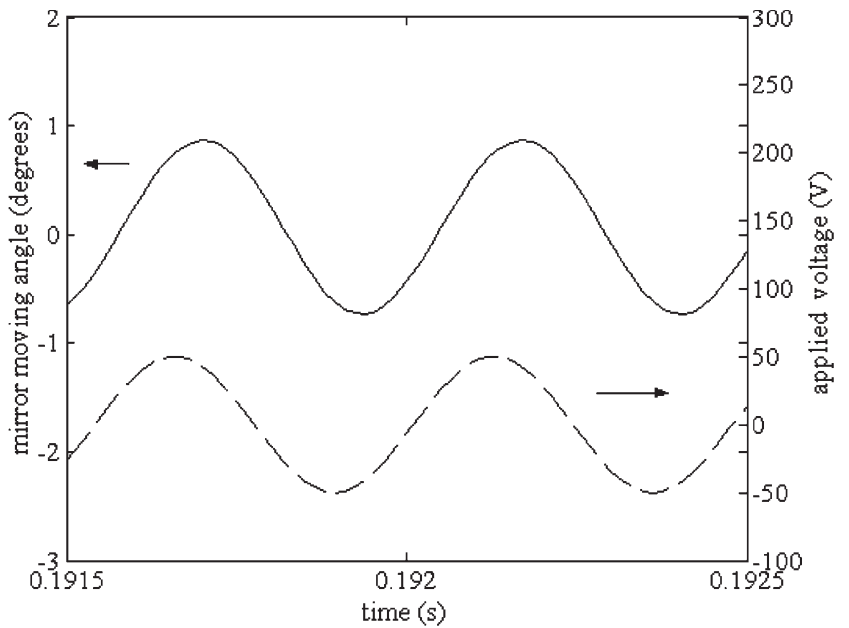

(a)

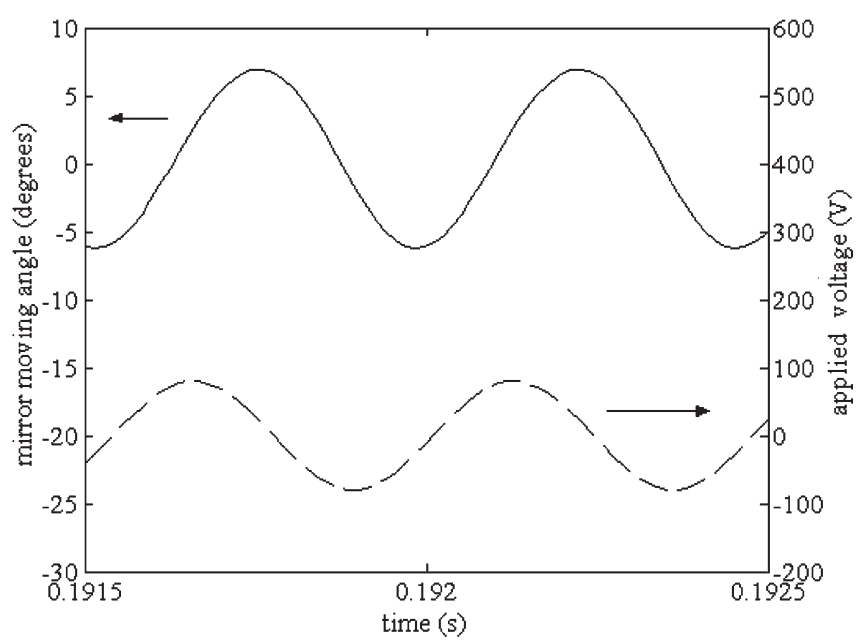

(b)

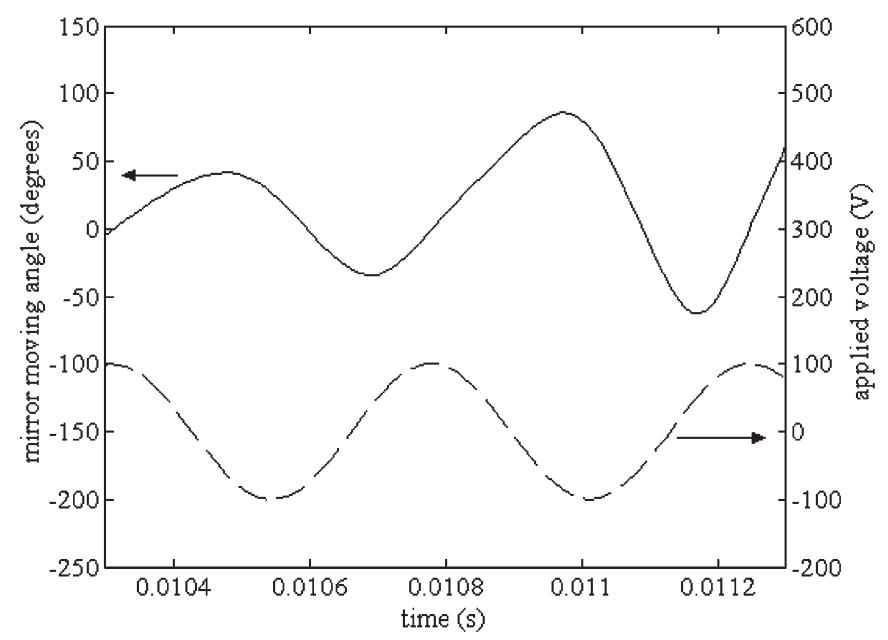

(c)

Fig. 5. Detailed simulated oscillation curves obtained with an applied voltage of (a) $50 \mathrm{~V}_{\mathrm{p}-\mathrm{p}}$, (b) $80 \mathrm{~V}_{\mathrm{p}-\mathrm{p}}$, and (c) $100 \mathrm{~V}_{\mathrm{p}-\mathrm{p}}$.

experimental result for the highest voltage. Fig. 6(b) shows the result obtained using a second-order approximation. The result is now much closer to the experiment, and the distortion in the response at high voltage can also be seen. Approximation to higher order in $\theta$ is not necessary, as can be seen from the $R^{2}$ 


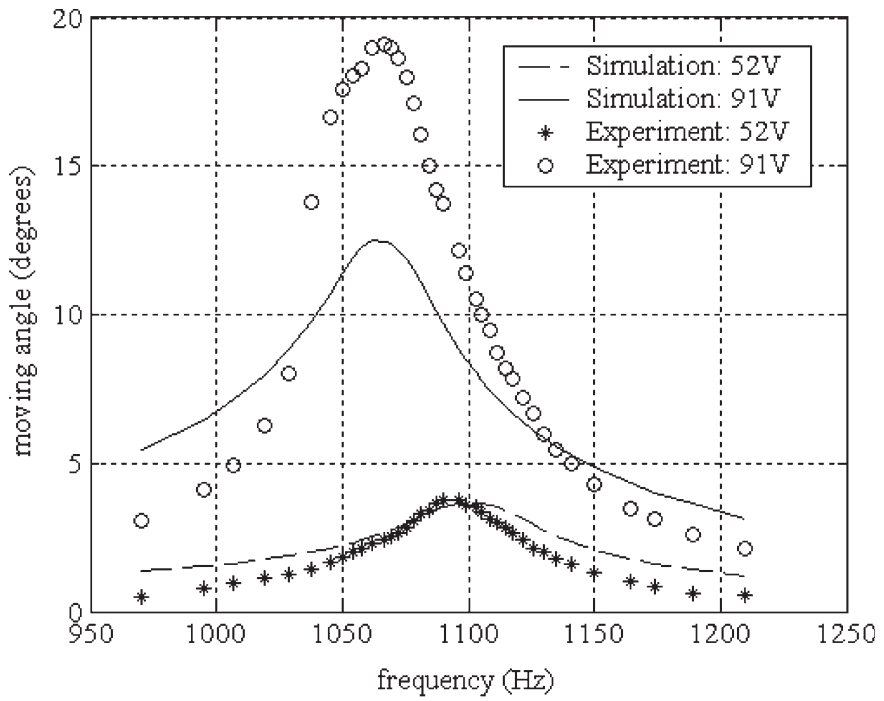

(a)

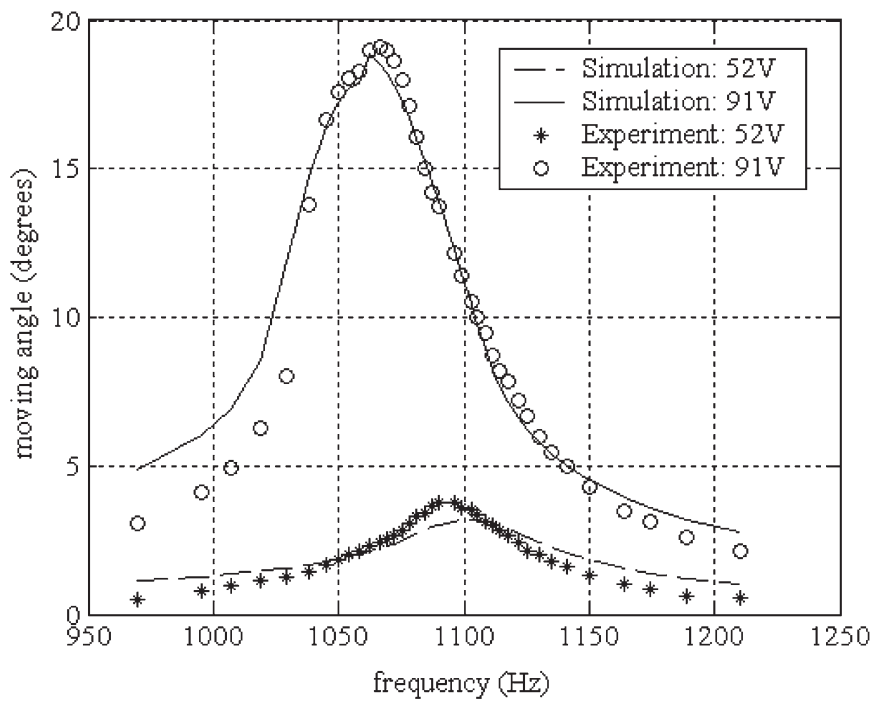

(b)

Fig. 6. Numerically simulated relation between frequency and angle obtained using (a) only the coefficients $c_{0}$ and $c_{1}$ in (4) and (b) coefficients $c_{0}, c_{1}$, and $c_{2}$.

TABLE I

Coefficients of the Power Series Variation of the Capacitance OBTAINED FROM NUMERICAL SIMULATION

\begin{tabular}{|c|c|c|c|c|c|c|}
\hline $\begin{array}{c}\text { Approximation } \\
\text { for capacitance }\end{array}$ & $\begin{array}{c}c_{0} \\
\left(\times 10^{-13}\right)\end{array}$ & $\begin{array}{c}c_{1} \\
\left(\times 10^{-13}\right)\end{array}$ & $\begin{array}{c}c_{2} \\
\left(\times 10^{-13}\right)\end{array}$ & $\begin{array}{c}c_{3} \\
\left(\times 10^{-13}\right)\end{array}$ & $\begin{array}{c}R^{2} \\
(52 \mathrm{~V})\end{array}$ & $\begin{array}{c}R^{2} \\
(91 \mathrm{~V})\end{array}$ \\
\hline $1^{\text {st }}$ order & 0.25 & 5.35 & & & 0.700 & 0.591 \\
\hline $2^{\text {nd }}$ order & 0.21 & 4.79 & 43.3 & & 0.826 & 0.969 \\
\hline $3^{\text {rd }}$ order & 0.21 & 4.79 & 43.3 & 0.02 & 0.826 & 0.969 \\
\hline
\end{tabular}

value in Table I. The mirror actuation torque can therefore be expressed as

$T(\theta)=1 / 2 \partial C / \partial \theta V^{2}=\left(0.11+2.39 \theta+21.7 \theta^{2}\right) \times 10^{-13} V^{2}$.

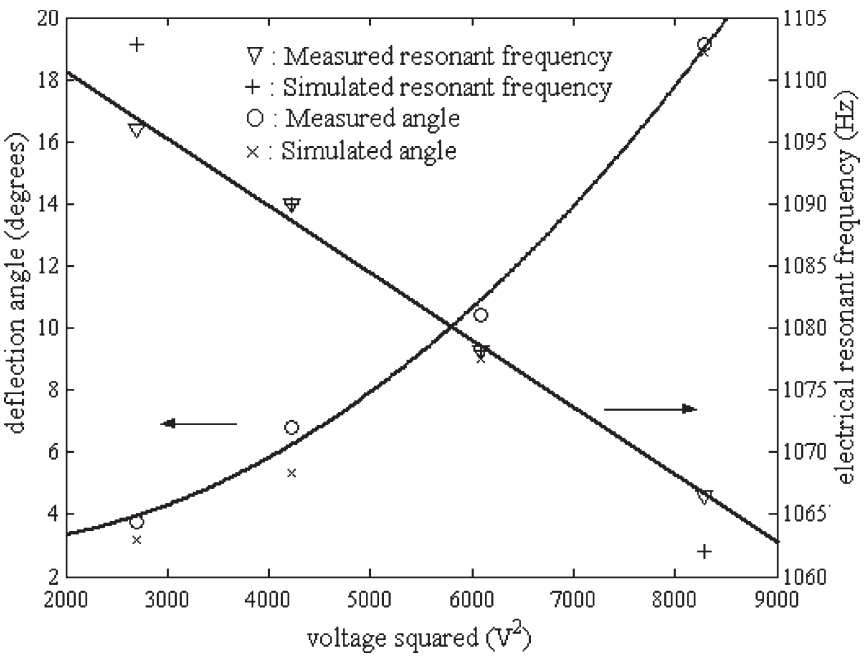

Fig. 7. Comparison of the predicted peak deflection and electrical resonance frequency with the measured data.

The solution provides a good fit for the nonlinear behavior seen at high voltage up to $97 \%$, whereas for low voltage, the predicted result fits up to $83 \%$. This difference may result from measurement error. Fig. 7 shows the predicted peak deflection for two other applied voltages of 65 and $78 \mathrm{~V}$ obtained using (13). The simulated results again agree well with the experiment. Although the size of the higher order terms appears surprising, the explanation is simply that the effect of interdigitation of the electrode system is largely lost at the rest position of the moving electrode, and as a result, the system shows some of the strong nonlinearity of a parallel-plate drive.

\section{CCR RESPONSE}

Using a model that has now been validated for the steadystate response, the transient response (Fig. 3) may be predicted, and the power transmitted to the receiver may be simulated. The calculation starts with ray tracing through the CCR. Fig. 8(a) shows a ray trace through a perfectly aligned system [14]. When an incident ray along the incident direction $-\tilde{n}_{i}$ strikes the CCR, the ray is reflected from each of the three mirrors in turn and exits along the direction $\tilde{n}_{i}$. The effective area can be defined as the area within which a ray can strike all three mirrors and still be reflected back to the light source. Fig. 8(b) shows the effective area for the case where $2 n_{i x} \geq n_{i z} \geq$ $n_{i y} \geq n_{i x}$. Since the incident rays generally strike the CCR along the body diagonal direction, we can use the effective area in Fig. 8(b) for the simulation.

To calculate the power transmitted to the receiver, Zhu et al. used the differential scattering cross section (DSCS), which is defined as the reflected power per unit solid angle of observation per unit illumination irradiance or $d \sigma\left(\tilde{n}_{i}, \tilde{n}_{o}\right) / d \Omega_{o}$. Here, $\tilde{n}_{o}$ is the reflected beam direction, and $\Omega_{o}$ is the solid angle of observation. The received power $P_{r}$ can then be expressed as [1]

$$
P_{r}=I_{i} r_{m}^{3} \times \int_{\Omega_{0} \in \Omega_{r}}\left\{d \sigma\left(\tilde{n}_{i}, \tilde{n}_{o}\right) / d \Omega_{o}\right\} d \Omega_{o} .
$$




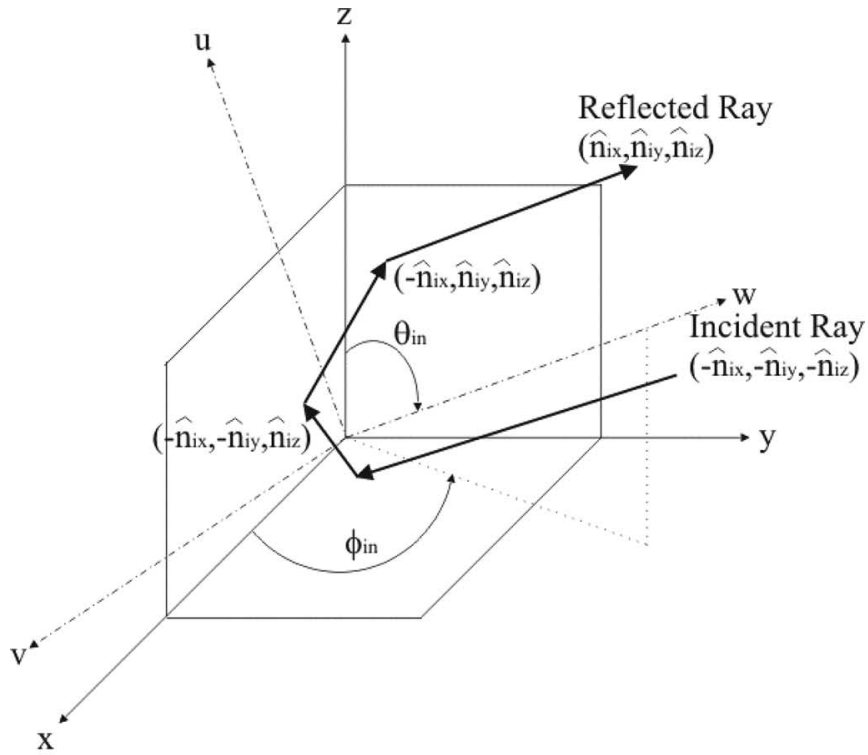

(a)

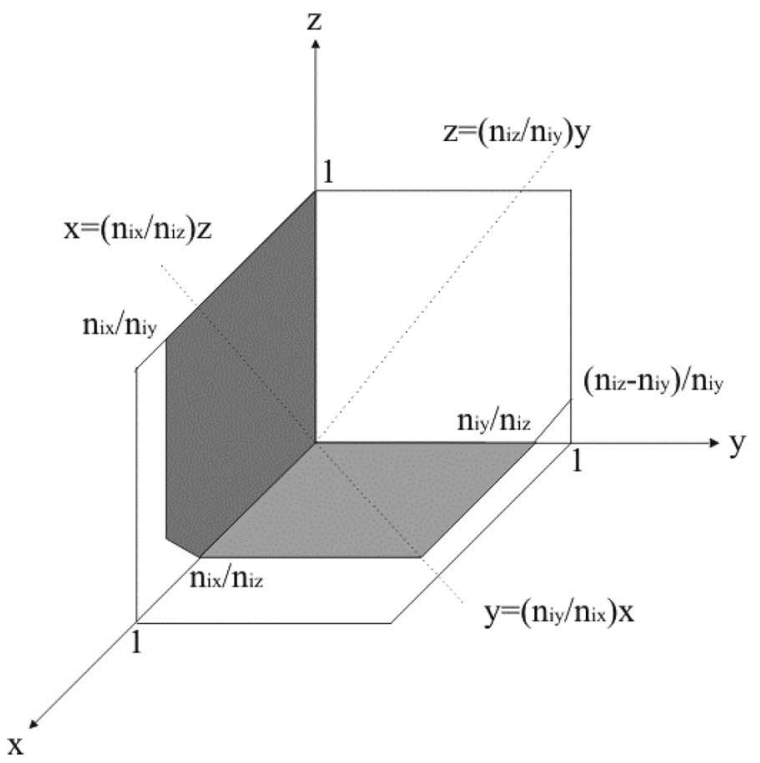

(b)

Fig. 8. Optical model of the CCR: (a) ray tracing and (b) effective area.

Here, $I_{i}$ is the light intensity incident on the CCR, $r_{m}$ is the reflectivity of the mirror, and $\Omega_{r}$ is the solid angle subtended by the receiver. When the direction of illumination and observation are colinear, $\tilde{n}_{o}=\tilde{n}_{i}$. This assumption is reasonable because the receiver is usually placed along the axis of illumination in a CCR link system, and the distance to the receiver is much larger than its diameter. The colinear DSCS (CDSCS) can therefore be expressed approximately as [14]

$$
P_{r}=I_{i} r_{m}^{3}\left\{d \sigma\left(\tilde{n}_{i}, \tilde{n}_{i}\right) / d \Omega_{o}\right\} \Omega_{r} .
$$

The power detected at the receiver then can be modeled by calculating the DSCS. The DSCS can be expressed using an optical relation as

$$
d \sigma\left(\tilde{n}_{i}, \tilde{n}_{o}\right) / d \Omega_{o}=I_{0} R^{2} / I_{i}=\left|E_{0}\left(\tilde{n}_{i}, \tilde{n}_{o}\right)\right|^{2} R^{2} / 2 I_{i} .
$$

Here, $R$ is the distance between the receiver and the CCR.

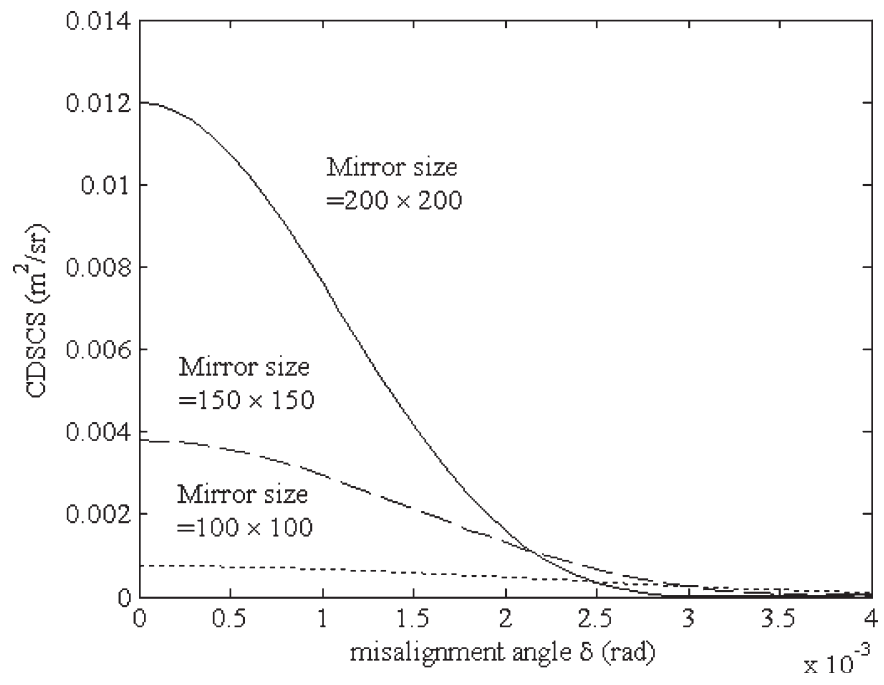

Fig. 9. Variation of CDSCS with misalignment angle $\delta$ for different CCR mirror sizes.

During modulation, when one of the mirrors is misaligned by an angle $\delta$, the electric field amplitude at the receiver position $E_{0}\left(\tilde{n}_{i}, \tilde{n}_{o}\right)$ is altered. Assuming that the mirrors are perfectly flat (a reasonable assumption for BSOI material), we can use a relatively simple relation for the electric field amplitude modified from [14] as

$$
\begin{aligned}
& E_{0}\left(\tilde{n}_{i}, \tilde{n}_{o}\right)=\left[\sqrt{ }\left(2 I_{i}\right) \exp \right.\{-\mathrm{j}(k d+\pi \nu)\} / \lambda R] \\
& \times \iint_{S_{u v}} \exp \left\{-\mathrm{j} \psi_{u v}(u, v) d u d v .\right.
\end{aligned}
$$

Here, $k=2 \pi / \lambda, \lambda$ is the wavelength of the light $(6328 \AA$ for a HeNe laser), $d$ is the path traveled from source to receiver, $\nu$ is the number of reflections undergone, the $u-v$ plane is the plane perpendicular to the incident beam direction $\tilde{n}_{i}, S_{u v}$ is the total effective area, and $\psi_{u v}(u, v)$ is the phase delay of a beam at the $(u, v)$ plane. Assuming only one mirror (for example, the $x y$ mirror) is modulated, the phase delay $\psi_{u v}(u, v)$ can be expressed as

$$
\psi_{u v}(u, v)=\psi_{x y}(x, y)=2 k \cdot \cos \left[\theta\left(\tilde{n}_{x y}, \tilde{n}_{i}\right)\right] \cdot y \cdot \delta_{y} .
$$

Here, $\delta_{y}$ is the $y$ component of the deviation after the mirror has rotated through the angle $\delta$, and $\tilde{n}_{x y}$ is the normal vector of the rotated $x y$ mirror plane. The CDSCS can be simulated using (16)-(18). Fig. 9 shows the predicted variation of CDSCS with misalignment angle $\delta$ for different mirror sizes. The incident direction is set along the body diagonal $\tilde{n}_{i}=(1 / \sqrt{ } 3,1 / \sqrt{ } 3,1 / \sqrt{ } 3)$, which is reasonable for the link where the distance from the light source to the CCR is large. Fig. 9 shows that the CDSCS angle increases as the mirror size increases and that a small misalignment of $3 \mathrm{mrad}$ is enough to switch the CCR from ON-state to OFF-state. This result is also consistent with the literature [14].

Dynamic behavior can then be modeled by substituting the mirror response obtained in the previous section into the 


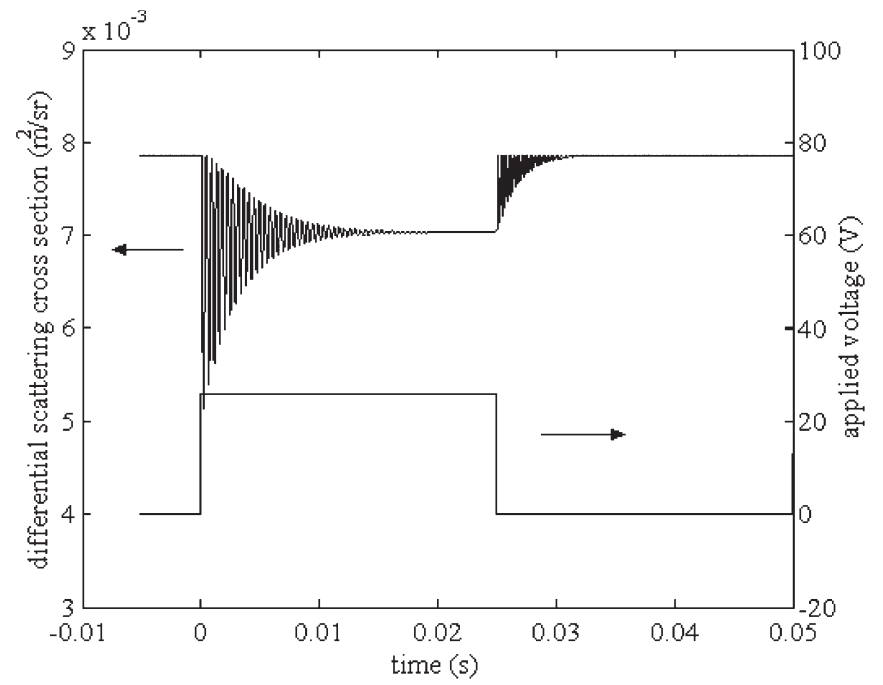

(a)

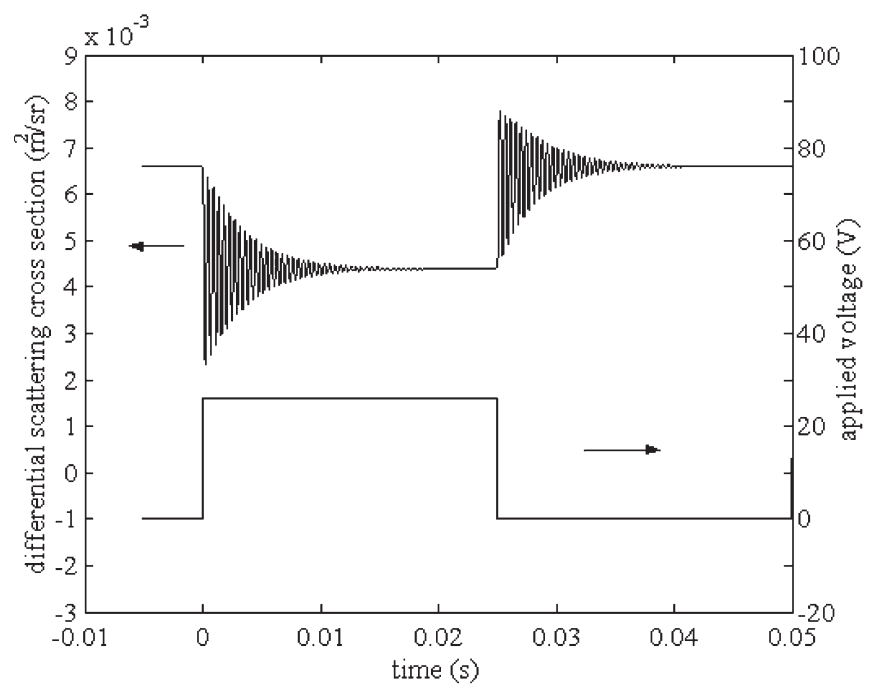

(b)

Fig. 10. Predicted dynamic response of a CCR actuated by a 20-Hz square wave with a voltage of $26 \mathrm{~V}_{\mathrm{p}-\mathrm{p}}$ (a) for an ideal case and (b) for a misalignment of $1 \mathrm{mrad}$.

misalignment term of this CCR model. In the experiment, the actuation voltage is a square wave with a frequency of $20 \mathrm{~Hz}$ at different voltages. Fig. 10 shows the predicted dynamic response at a low voltage $\left(26 \mathrm{~V}_{\mathrm{p}-\mathrm{p}}\right)$. Fig. 10(a) shows the DSCS obtained in the ideal case, when all the three mirrors are aligned perfectly. This result should be compared with the experimental result previously shown in Fig. 3(a). Ideally, the DSCS has the maximum value when the mirror angle is zero. The signal at the beginning of turn-off shows a beating, which corresponds to the mirrors oscillating in the opposite direction. The experimental result, however, shows higher value at the beginning of the turn-off state and no beating. This result implies that the CCR initially must have a small angular misalignment. Fig. 10(b) shows the DSCS, assuming an initial misalignment of 1 mrad. In this case, the predicted DSCS shape is consistent with the experimental data.

The dynamic response at a high voltage can also be predicted. Fig. 11(a) and (b) shows the response at $48 \mathrm{~V}_{\mathrm{p}-\mathrm{p}}$ without and

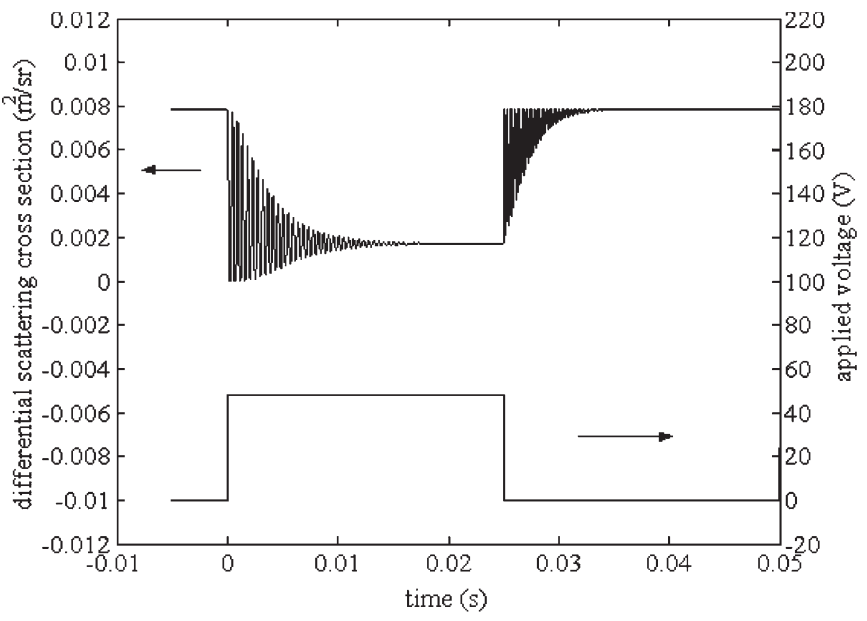

(a)

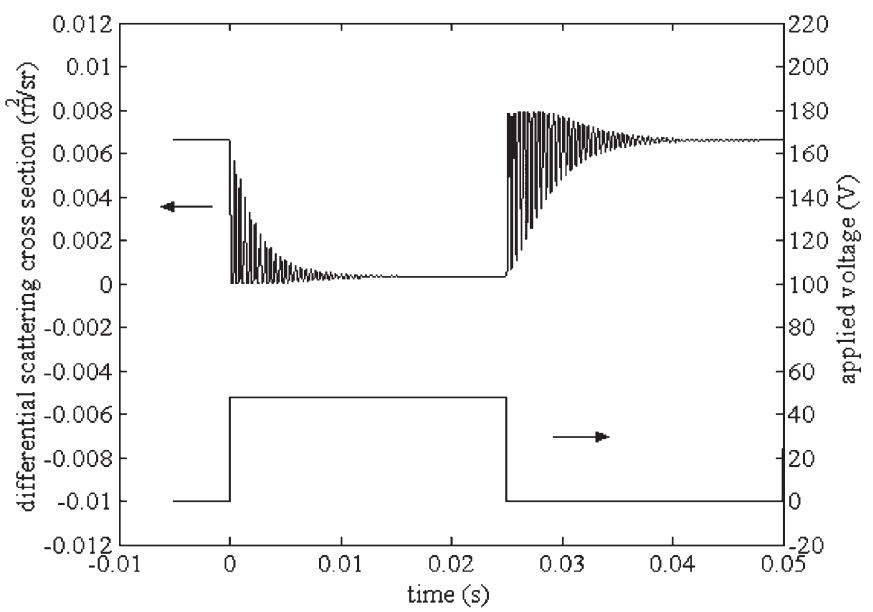

(b)

Fig. 11. Predicted dynamic response of a CCR actuated by a $20-\mathrm{Hz}$ square wave with a voltage of $48 \mathrm{~V}_{\mathrm{p}-\mathrm{p}}$ (a) for an ideal case and (b) for a misalignment of $1 \mathrm{mrad}$.

with a misalignment, respectively. In this case, when the voltage is turned on, the deviation of the CCR mirror is sufficient to cause the retro-reflected beam to miss the detector entirely. This effect causes a "clipping" of the DSCS, as can be seen in Fig. 11(a). Fig. 11(b) shows the DSCS, assuming an initial misalignment of $1 \mathrm{mrad}$. Because the oscillation angle of the mirror at the beginning of the turn-off state exceeds the initial misalignment, some beating oscillations can be seen in Fig. 11(b). This result also coincides with the experimental data previously shown in Fig. 3(b).

Although the model suggested in this section is well matched with the experimental data, the predicted and experimental results do not agree exactly. The reason lies in the geometry of the experimental CCR, which has a larger overall area than the three perpendicular mirrors. For example, there is a land between the two $45^{\circ}$ mirrors, as shown in Fig. 1(b). This region affects the ray trace reflected from the mirrors. In addition, the $90^{\circ}$ mirror has a different size and shape compared to the $45^{\circ}$ mirrors. Finally, actuation is also different in the real device. We have assumed, for simplicity, that only one mirror was actuated in the simulation. In fact, two mirrors are driven 
experimentally. These differences could be easily resolved in an extended model.

\section{CONCLUSION}

A model for the dynamic response of an electrostatic micromirror driven by an angular vertical combdrive actuator has been proposed based on a numerical solution of the nonlinear equations of motion for a torsional oscillator. The model has been compared with experimental data obtained from selfassembled micromirror devices. From the solution, it has been shown that the torque generated by the angular vertical drive depends at least on the second order of the turn angle, which results in a deformation in the shape of the resonance curve at high voltage. The numerical solution also shows a shift in the resonant frequency with voltage, and the model can accurately predict the resonant frequency at an arbitrary input voltage. However, simpler models for the capacitance variation do not provide sufficient accuracy.

By extending the model, the dynamic response of a CCR based on similar micromirrors can be predicted. The theoretical predictions agree well with the experimental results. From the model, a small initial angular misalignment of the CCR mirrors can also be determined. The model highlights the high assembly accuracy required in a practical CCR system.

\section{REFERENCES}

[1] L. Zhou, J. M. Kahn, and K. S. J. Pister, "Corner-cube retroreflectors based on structure-assisted assembly for free-space optical communication," J. Microelectromech. Syst., vol. 12, no. 3, pp. 233-242, Jun. 2003.

[2] V. Milanovic, S. Kwon, and L. P. Lee, "High aspect ratio micromirrors with large static rotation and piston actuation," IEEE Photon. Technol. Lett., vol. 16, no. 8, pp. 1891-1893, Aug. 2004.

[3] A. Selvakumar and K. Najafi, "Vertical comb array microactuators," J. Microelectromech. Syst., vol. 12, no. 4, pp. 440-449, Aug. 2003.

[4] D. Hah, S. T. Huang, J. C. Tsai, H. Toshiyoshi, and M. C. Wu, "Lowvoltage, large-scan angle MEMS analog micromirror arrays with hidden vertical comb-drive actuators," J. Microelectromech. Syst., vol. 13, no. 2, pp. 279-289, Apr. 2004.

[5] P. R. Patterson, D. Hah, H. Nguyen, H. Toshiyoshi, R. Chao, and M. C. Wu, "A scanning micromirror with angular comb drive actuation," in Proc. IEEE 15th Int. Conf. Micro Electro Mech. Syst., Las Vegas, NV, Jan. 20-24, 2002, pp. 544-547.

[6] J. A. Yeh, H. Jiang, and N. C. Tien, "Integrated polysilicon and DRIE bulk silicon micromachining for an electrostatic torsional actuator," J. Microelectromech. Syst., vol. 8, no. 4, pp. 456-465, Dec. 1999.

[7] R. R. A. Syms, "Surface tension powered self-assembly of 3-D microoptomechanical structures," J. Microelectromech. Syst., vol. 8, no. 4, pp. 448-455, Dec. 1999.

[8] D. Hah, P. R. Patterson, H. D. Nguyen, H. Toshiyoshi, and M. C. Wu, "Theory and experiment of angular vertical comb-drive actuators for scanning micromirrors," IEEE J. Sel. Topics Quantum Electron., vol. 10, no. 3, pp. 505-513, May/Jun. 2004.
[9] Y. K. Hong, R. R. A. Syms, K. S. J. Pister, and L. X. Zhou, "Design, fabrication and test of self-assembled optical corner cube reflectors," J. Micromech. Microeng., vol. 15, no. 3, pp. 663-672, Mar. 2005.

[10] R. R. A. Syms, "Self-assembled 3-D silicon microscanners with selfassembled electrostatic drives," IEEE Photon. Technol. Lett., vol. 12, no. 11, pp. 1519-1521, Nov. 2000.

[11] J. L. A. Yeh, C. Y. Hui, and N. C. Tien, "Electrostatic model for an asymmetric combdrive," J. Microelectromech. Syst., vol. 9, no. 1, pp. 126135, Mar. 2000

[12] H. Xie, Y. Pan, and G. K. Fedder, "A CMOS-MEMS mirror with curledhinge comb drives," J. Microelectromech. Syst., vol. 12, no. 4, pp. 450457, Aug. 2003.

[13] R. R. A. Syms, C. Gormley, and S. Blackstone, "Improving yield, accuracy and complexity in surface tension self-assembled MOEMS," Sens. Actuators A, Phys., vol. 88, no. 3, pp. 273-283, Jan. 2001.

[14] X. Zhu, V. S. Hsu, and J. M. Kahn, "Optical modelling of MEMS corner cube retro reflectors with misalignment and nonflatness," IEEE J. Sel. Topics Quantum Electron., vol. 8, no. 1, pp. 26-32, Jan./Feb. 2002.

[15] E. Kreyszig, Advanced Engineering Mathematics, 3rd ed. New York: Wiley, 1972, pp. 665-667.

[16] J. R. Dormand and P. J. Prince, "A family of embedded Runge-Kutta formulae," J. Comput. Appl. Math., vol. 6, no. 1, pp. 19-26, 1980.

[17] W. T. Thomson and M. D. Dahleh, Theory of Vibration With Applications, 5th ed. Englewood Cliffs, NJ: Prentice-Hall, 1998, ch. 2/3.

[18] W. Mentenhall and T. Sincich, Statistics for Engineering and the Sciences, 4th ed. Englewood Cliffs, NJ: Prentice-Hall, 1995, pp. 566-567.

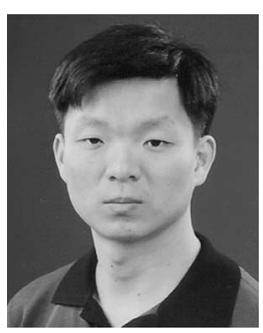

Young Ki Hong was born in Seoul, Korea, in 1970. He received the M.S. degree in physics from Yonsei University, Seoul, in 1994 and the Ph.D. degree from Imperial College London, London, U.K., in 2006.

He was with the Samsung Semiconductor R\&D Institution, Seoul, for eight years before moving to the Optical and Semiconductor Devices Group, Department of Electrical and Electronic Engineering, Imperial College London. He is currently with the Samsung Semiconductor R\&D Institution. His research interests include optical MEMS devices, surface micromachining, and material characterization.

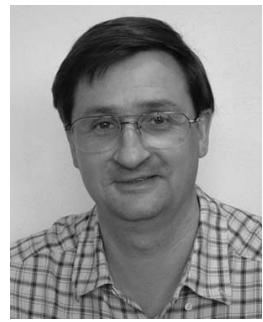

Richard R. A. Syms (M'98-SM'02) was born in Norfolk, VA, in 1958. He received the B.A. degree in engineering science and the D.Phil. degree (on volume holographic optical elements) from Worcester College, Oxford, U.K., in 1979 and 1982, respectively.

He has been the Head of the Optical and Semiconductor Devices Group, Department of Electrical and Electronic Engineering, Imperial College London, London, U.K., since 1992 and a Professor of microsystems technology since 1996. He is also a Cofounder and the Research Director of the MEMS spin-off company Microsaic Systems. He has published more than 100 journal papers and two books on holography, integrated optics, laser and amplifier devices, and microengineering.

Prof. Syms currently acts as an Associate Editor for the IEEE/ASME Journal of Microelectromechanical Systems. He is a Fellow of the Institution of Electrical Engineers, the Institute of Physics, and the Royal Academy of Engineering. 